\title{
CORRECTION
}

\section{Correction to: Characterization of Poultry Litter Ash in View of Its Valorization}

\author{
L. Luyckx ${ }^{1}$. $\cdot$ G. H. J. de Leeuw ${ }^{2} \cdot$ J. Van Caneghem ${ }^{1}$
}

Published online: 31 July 2019

○) Springer Nature B.V. 2019

\section{Correction to: Waste and Biomass Valorization https://doi.org/10.1007/s12649-019-00750-6}

Unfortunately, Table 1 was published incorrectly in the original publication of the article. The correct version of Table 1 is provided here.

Publisher's Note Springer Nature remains neutral with regard to jurisdictional claims in published maps and institutional affiliations.

The original article can be found online at https://doi.org/10.1007/ s12649-019-00750-6.

L. Luyckx

lorien.luyckx@kuleuven.be

1 Materials Technology TC, KU Leuven, Campus Group

T, Andreas Vesaliusstraat 13 - box 2600, Leuven 3000,

Belgium

2 BMC Moerdijk BV, Industrial Park M349, Middenweg 36a, 4782 PM Moerdijk, The Netherlands 
Table 1 Average

concentration \pm standard deviation of $\mathrm{P}, \mathrm{K}, \mathrm{Ca}, \mathrm{Mg}, \mathrm{Na}$, $\mathrm{S}, \mathrm{Si}, \mathrm{Al}, \mathrm{Cd}, \mathrm{Cr}, \mathrm{Cu}, \mathrm{Fe}, \mathrm{Mn}$, $\mathrm{Mo}, \mathrm{Ni}, \mathrm{Pb}, \mathrm{Ti}$ and $\mathrm{Zn}$ in the bed, SH, ECO, ESP, bag and mixed ash (\% of DM and $\mathrm{mg} / \mathrm{kg}$ $\mathrm{DM}) ; \mathrm{n}=3$

\begin{tabular}{llccccc}
\hline & Bed ash & \multicolumn{1}{c}{ SH ash } & \multicolumn{1}{c}{ ECO ash } & \multicolumn{1}{c}{ ESP ash } & \multicolumn{1}{c}{ Bag ash } & Mixed ash \\
\hline $\mathrm{P}(\%$ of DM) & $5.0 \pm 0.03$ & $10.6 \pm 0.11$ & $8.9 \pm 0.10$ & $6.4 \pm 0.06$ & $2.3 \pm 0.32$ & $6.0 \pm 0.08$ \\
$\mathrm{~K}(\%$ of DM) & $9.4 \pm 0.07$ & $11.1 \pm 0.06$ & $11.5 \pm 0.07$ & $15.5 \pm 1.32$ & $14.3 \pm 1.55$ & $12.5 \pm 0.30$ \\
$\mathrm{Ca}(\%$ of DM$)$ & $20.2 \pm 0.10$ & $19.3 \pm 0.11$ & $20.1 \pm 0.22$ & $12.4 \pm 0.08$ & $20.6 \pm 1.60$ & $18.2 \pm 0.36$ \\
$\mathrm{Mg}(\%$ of DM$)$ & $2.6 \pm 0.03$ & $6.2 \pm 0.06$ & $5.2 \pm 0.04$ & $3.5 \pm 0.01$ & $1.2 \pm 0.15$ & $3.0 \pm 0.56$ \\
$\mathrm{Na}(\%$ of DM$)$ & $2.7 \pm 0.02$ & $2.6 \pm 0.02$ & $3.4 \pm 0.01$ & $3.6 \pm 0.02$ & $1.6 \pm 0.12$ & $1.9 \pm 0.02$ \\
$\mathrm{~S}(\%$ of DM) & $3.1 \pm 0.02$ & $1.7 \pm 0.05$ & $1.8 \pm 0.02$ & $6.0 \pm 0.05$ & $4.8 \pm 0.48$ & $3.1 \pm 0.03$ \\
$\mathrm{Si}(\%$ of DM) & $9.2 \pm 0.39$ & $4.0 \pm 0.03$ & $5.6 \pm 0.05$ & $2.8 \pm 0.04$ & $1.4 \pm 0.12$ & $6.8 \pm 0.27$ \\
$\mathrm{Al}(\mathrm{mg} / \mathrm{kg} \mathrm{DM})$ & $6642 \pm 113$ & $7799 \pm 84$ & $9306 \pm 69$ & $5671 \pm 42$ & $2494 \pm 69$ & $5021 \pm 187$ \\
$\mathrm{Cd}(\mathrm{mg} / \mathrm{kg} \mathrm{DM})$ & $<2$ & $3.5 \pm 0.2$ & $2.7 \pm 0.2$ & $4.9 \pm 0.3$ & $3.1 \pm 0.07$ & $2.1 \pm 0.02$ \\
$\mathrm{Cr}(\mathrm{mg} / \mathrm{kg} \mathrm{DM})$ & $15.9 \pm 0.5$ & $29.7 \pm 1.6$ & $29.2 \pm 0.8$ & $31.2 \pm 0.8$ & $16.1 \pm 0.6$ & $18.7 \pm 0.2$ \\
$\mathrm{Cu}(\mathrm{mg} / \mathrm{kg} \mathrm{DM})$ & $391 \pm 6$ & $741 \pm 49$ & $568 \pm 31$ & $698 \pm 36$ & $353 \pm 8$ & $435 \pm 6$ \\
$\mathrm{Fe}(\mathrm{mg} / \mathrm{kg} \mathrm{DM})$ & $4423 \pm 204$ & $6787 \pm 85$ & $8168 \pm 326$ & $4444 \pm 53$ & $2216 \pm 32$ & $4284 \pm 205$ \\
$\mathrm{Mn}(\mathrm{mg} / \mathrm{kg} \mathrm{DM})$ & $1595 \pm 20$ & $3594 \pm 15$ & $3076 \pm 47$ & $2090 \pm 17$ & $761 \pm 16$ & $1939 \pm 16$ \\
$\mathrm{Mo}(\mathrm{mg} / \mathrm{kg} \mathrm{DM})$ & $15.0 \pm 0.5$ & $24.1 \pm 1.1$ & $19.9 \pm 1.0$ & $33.5 \pm 2.4$ & $22.8 \pm 0.7$ & $18.0 \pm 0.1$ \\
$\mathrm{Ni}(\mathrm{mg} / \mathrm{kg} \mathrm{DM})$ & $23.1 \pm 0.6$ & $46.4 \pm 2.5$ & $36.6 \pm 1.0$ & $33.7 \pm 3.0$ & $10.9 \pm 0.3$ & $25.2 \pm 0.5$ \\
$\mathrm{~Pb}(\mathrm{mg} / \mathrm{kg} \mathrm{DM})$ & $<2$ & $8.0 \pm 0.9$ & $5.2 \pm 0.1$ & $13.6 \pm 0.7$ & $10.1 \pm 0.5$ & $4.5 \pm 1.0$ \\
$\mathrm{Ti}(\mathrm{mg} / \mathrm{kg} \mathrm{DM})$ & $226 \pm 6$ & $464 \pm 27$ & $514 \pm 24$ & $342 \pm 22$ & $121 \pm 3$ & $241 \pm 1$ \\
$\mathrm{Zn}(\mathrm{mg} / \mathrm{kg} \mathrm{DM})$ & $985 \pm 32$ & $2226 \pm 99$ & $1755 \pm 68$ & $2591 \pm 205$ & $1150 \pm 19$ & $1497 \pm 39$ \\
\hline
\end{tabular}

